- Original Article

\title{
Cancer Prevalence among Physicians in Korea: A Single Center Study
}

\author{
Hye Lin Kim*, Hae Jin Park, Yun Hye Sim, Eun Young Choi, Kyung Won Shim, Sang Wha Lee, Hong Soo Lee, \\ Hyejin Chun
}

Department of Family Medicine, Ewha Womans University Mokdong Hospital, Seoul, Korea

Background: There is little research regarding whether working as a physician affects cancer risk. Moreover, there is no research on cancer prevalence among physicians in Korea. This study utilized the Korea National Cancer Incidence Database to determine whether the prevalence of cancer among physicians differs from the prevalence of cancer within the general population.

Methods: We analyzed the medical records of a representative sample of 382 doctors who underwent a health examination between 2010 and 2013 at a health examination center in a Ewha Womans University Medical Center. Cancer incidence was measured as cases that were eventually diagnosed as cancer according to a biopsy.

Results: We collected medical records from 382 physicians (mean age, 51.9 \pm 8.1 years) and calculated the standardized prevalence ratios compared to the general population. Thirty physicians ( 9 male and 21 female) were identified as having cancer. Physicians had a significantly higher prevalence of cancer compared to the general population. Cancer prevalence in male physicians was found to be 2.47 times higher than the prevalence expected within the general population $(\mathrm{P}=0.006)$. Among female physicians, cancer prevalence was 3.94 times higher than that in the general population $(\mathrm{P}<0.001)$.

Conclusion: This study revealed that physicians had a higher prevalence of cancer compared to the general population in Korea, which suggests that there may be a problem present in the health care of physicians. Changes to the working environment of physicians will be needed to reduce the high prevalence of cancer among physicians.

Keywords: Physicians; Cancer; Prevalence; Korea 


\section{INTRODUCTION}

As might be expected, physicians are reported to have healthier lifestyles and lower mortality rates than the general population as a result of their professional medical knowledge. ${ }^{1-3)}$ However, in some studies, the actual mortality rate among physicians differed according to specialty, while in other studies, no significant differences in mortality rates were found between specialties. ${ }^{4,5)}$ There are reports suggesting that since physicians have easier access to medical care compared to others, as well as healthier lifestyles, they can better educate their patients. ${ }^{1,3)}$ As such, a physician's knowledge and experience will affect the advice and treatment they give to their patients. In contrast, there are several studies stating that physicians do not take care of their own health any better than expected. ${ }^{4,-8)}$ Some studies report that physicians actually get fewer vaccinations, are careless about controlling their cardiovascular risk factors, and receive fewer screening tests for cancer than the general population. More so, some studies suggest that about half of physicians do not have anyone to consult regarding their own medical problems. ${ }^{9,10)}$

A case report of one physician in Australia described how he determined his symptoms on his own and how he was later diagnosed with lymphoma. ${ }^{11)}$ In that report, he asserted that at some point, most physicians will live as a patient 'on the other side.' He also confessed that he was not familiar with the role of 'patient.' Although he is now in remission, the experience of being a patient gave him a lesson in how to care for his own patients as a physician. The important points from this report are (1) physicians may develop a disease, (2) physicians do not always receive good medical advice and are not always familiar with appropriate medical treatments outside of their own specialties, and (3) finding their own health care provider is important, even if the physician is not sick now.

Physicians are exposed to various occupational and environmental factors that may increase the risk of cancer despite their medical knowledge. Physicians are part of a unique group of individuals who are routinely exposed to multiple carcinogens, such as ionizing radiation and various chemicals. Exposure to high doses of diagnostic and therapeutic ionizing radiation is known to increase the risk of various cancers. ${ }^{12-15)}$ Moreover, although there are differences depending on the specialty, physicians are reported to experience a great deal of physical and mental stress. ${ }^{16,17)}$ Stress caused by a heavy workload, burnout syndrome, compassion fatigue, and short sleep can cause many adverse effects, including cancer. ${ }^{18,19)}$ Furthermore, Kang et al. ${ }^{16)}$ reported that most surgeons (82.5\%) did not recommend that their children follow in their professional footsteps. We hypothesized that these factors could increase the risk of cancer in physicians.

Although there are some reports suggesting that physicians have risk factors for cancer development, no studies have been conducted on Korean physicians. Therefore, there is a strong need for research on physicians' health, not only to assess the current status of their health but to also manage their health. To this end, we reviewed the medical records of physicians who work at a university hospital and who visited the health check-up center for a routine health assessment. We assessed the prevalence of various cancer types among physicians as compared to the general population and investigated the cancer prevalence rate to identify the current health status of physicians in Korea.

\section{METHODS}

\section{Study Population}

This retrospective cross-sectional study examined the prevalence of cancer among physicians in Korea and investigated whether the prevalence of cancer among physicians differed from the prevalence among the general population. Subjects included physicians who participated in a medical health check-up program at the health promotion center of Ewha Womans University School of Medicine between 2010 and 2013. A total of 401 physicians were registered as potential subjects. Nineteen physicians did not respond to the medical history questionnaire and were subsequently excluded from the study, because we could not confirm their past history of cancer. Of 401 potentially eligible physicians, 382 physicians were registered in this study.

\section{Study Methods}

We collected data retrospectively. Between July and August 2014, two physicians reviewed the medical records of the participants, including basic subject information, health screening results, and responses to a medical history questionnaire. The information reviewed included: (1) the hospital identification number of physicians, (2) English name initials, (3) sex, (4) date of birth, (5) height and weight, (6) professional specialties, (7) work setting (e.g., private clinic vs. university hospital), (8) past history of disease, (9) past surgical history, and (10) health examination results. Height was measured down to $0.1 \mathrm{~cm}$ while subjects wore a light gown, with their heels flat on the ground and their back straight. Weight was measured while subjects wore a light gown of less than $0.1 \mathrm{~kg}$. Body mass index (BMI) was defined as weight ( $\mathrm{kg}$ ) divided by the square of their height $(\mathrm{m})$. Health examination results included findings from physical examinations, blood tests, endoscopy, and radiologic imaging.

\section{Cancer Validation}

Information regarding past or current cancer diagnoses was retrieved via health examinations and self-reported responses to a medical history questionnaire. Through this process, a total of 31 cases of cancer were validated. According to results from the health check-ups conducted between 2010 and 2013, 13 (41.9\%) of the 31 cases of cancer were newly diagnosed. Cancer diagnoses were confirmed via pathology reports and radiologic findings. Based on responses to the medical history questionnaire administered during routine examinations, we were able to identify 17 (58.1\%) cases of cancer from self-reported medical records, because medical records can confirm $>98 \%$ of cancers reported on death certificates. ${ }^{20)}$ The medical history questionnaire used to assess past diagnoses of cancer was composed of the following questions: “Have you ever been diagnosed with cancer?", What 
type(s) of cancer have you been diagnosed with?", and "When were you diagnosed with cancer?"

\section{Statistical Analyses}

Data were expressed as the mean \pm standard deviation for continuous variables and as a number (percentage) for categorical variables. Work setting was categorized into two groups: private clinic and university hospital. Specialties were categorized into eight distinct groups consisting of various specialties: internal medicine (internal medicine, family medicine, pediatrics, rehabilitation, and neurology), surgery (general surgery, obstetrics, orthopedic surgery, ophthalmology, otolaryngology, neurosurgery, plastic surgery, urology, thoracic surgery, dermatology, emergency medicine, and dentistry), radiology (radiology and radiation oncology), anesthesiology, psychiatry, pathology, others (laboratory medicine and basic medicine), and unknown.

The standardized prevalence ratio (SPR) is defined as the ratio of the actual observed number of cancer cases among physicians divided by the expected number of cancer cases among physicians. The expected number of cancer cases was calculated by multiplying the number of physicians by the cancer prevalence rate in the general population according to the Korea National Cancer Incidence Database, which is the most recent national statistic regarding the prevalence of cancer in $2011{ }^{21)}$ We also calculated the SPRs for each type of cancer separately as well as all cancers collectively. The SPRs were calculated under the assumption that the subjects who were identified as not having cancer truly did not have any cancers in any part of the body and that they experienced the same cancer prevalence as would be expected among the general population in Korea. We assumed that the data would have a Poisson distribution; therefore, 95\% confidence intervals (CIs) for the SPRs were estimated using standard errors. PASW SPSS Statistics for Windows ver. 18.0 (SPSS Inc., Chicago, IL, USA) was used for the statistical analyses. We considered P-values of less than 0.05 to be statistically significant in all analyses.

\section{Ethics Statement}

This cross-sectional study was approved by the institutional review board of Ewha Womans Mokdong Hospital (IRB no.: EUMC 2015-02005-004).

\section{RESULTS}

\section{Subject Characteristics}

The demographic characteristics of physicians according to sex are presented in Table 1. Of the 382 physicians included in this study, 172 were male (45.0\%) and 210 were female (55\%). The mean age of study participants was $51.9 \pm 8.1$ years; for male physicians, the mean age was $52.7 \pm 7.9$ years, and for female physicians, the mean age was $50.6 \pm 10.5$ years. The mean BMI of males included in the study was $24.9 \pm 2.5 \mathrm{~kg} / \mathrm{m}^{2}$; the overweight rate was $36.3 \%$ and the obesity rate was $44.8 \%$, as defined by the World Health Organization Expert Obesity Classification for Asians. ${ }^{22)}$ Among males, 6 subjects (3.5\%) were classified with se- vere obesity (defined as a BMI greater than $30.0 \mathrm{~kg} / \mathrm{m}^{2}$ ). The mean BMI of female physicians was $22.1 \pm 2.6 \mathrm{~kg} / \mathrm{m}^{2}$; the overweight rate was $23.8 \%$, and the obesity rate was $11.9 \%$. Unlike male physicians, there were no subjects with severe obesity among female physicians. Women in our study were somewhat younger and more likely to have a normal BMI $\left(<23 \mathrm{~kg} / \mathrm{m}^{2}\right)$ compared to men. In the distribution according to working condition, 245 physicians (64.1\%) worked in private clinics, and 137 physicians (35.9\%) worked in university hospitals. Among the physicians in this study, the most common specialty was internal medicine (39.3\%), followed by surgery (38.7\%), anesthesiology (5.0\%), radiology (3.9\%), psychiatry (3.1\%), and pathology (1.3\%). The distribution of physicians by specialty showed a high distribution of males in surgery and a high distribution of females in internal medicine. Female physicians were more common in the specialties of radiology, anesthesiology, psychiatry, and pathology compared to male physicians.

\section{Cancer Prevalence}

Table 2 shows cancer prevalence based on results from the comprehensive medical assessments and medical records of 382 physicians who received a health screening examination at a university hospital center. A total of 30 physicians were identified as having cancer. However, one female physician had two cancers, of the stomach and colon.

Table 1. Baseline characteristics of 382 physicians according to sex

\begin{tabular}{lccc}
\hline \multicolumn{1}{c}{ Characteristic } & Male & Female & Total \\
\hline Total no. of subjects & $172(45.0)$ & $210(55.0)$ & $382(100.0)$ \\
Age (y) & & & \\
Mean age & $50.6 \pm 10.5$ & $52.7 \pm 7.9$ & $51.9 \pm 8.1$ \\
$<35$ & $1(0.6)$ & $6(2.9)$ & $7(1.8)$ \\
35-45 & $28(16.3)$ & $43(20.5)$ & $71(18.7)$ \\
45-55 & $68(39.5)$ & $86(41.2)$ & $154(40.3)$ \\
55-64 & $62(36.0)$ & $52(24.8)$ & $114(29.8)$ \\
$\geq 65$ & $13(7.6)$ & $23(10.6)$ & $36(9.4)$ \\
BMI (kg/m²) & & & \\
Mean BMl & $24.9 \pm 2.5$ & $22.1 \pm 2.6$ & $23.4 \pm 2.7$ \\
$<18.5$ & - & $14(6.7)$ & $14(3.7)$ \\
18.5-22.9 & $33(19.2)$ & $121(57.6)$ & $154(40.3)$ \\
23.0-24.9 & $62(36.0)$ & $50(23.8)$ & $112(29.3)$ \\
25.0-29.9 & $71(41.3)$ & $25(11.9)$ & $96(25.1)$ \\
$\geq 30.0$ & $6(3.5)$ & - & $6(1.6)$ \\
Specializations & & $16(7.6)$ & $21(5.5)$ \\
Internal medicine & $54(31.4)$ & $96(45.7)$ & $150(39.3)$ \\
Surgery & $95(55.2)$ & $53(25.2)$ & $148(38.7)$ \\
Radiology & $3(1.7)$ & $12(5.7)$ & $15(3.9)$ \\
Anesthesiology & $2(1.2)$ & $17(8.1)$ & $19(5.0)$ \\
Psychiatry & $4(2.3)$ & $8(3.8)$ & $12(3.1)$ \\
Pathology & $1(0.6)$ & $4(1.9)$ & $5(1.3)$ \\
Others & $5(2.9)$ & $135(64.3)$ & $245(64.1)$ \\
Unknown & $8(4.7)$ & & $137(35.9)$ \\
Work setting & & & \\
Private clinic & & & \\
University hospital & & & \\
\hline
\end{tabular}

Values are presented as number (\%) or mean \pm standard deviation. BMI, body mass index. 
Table 2. Cancer prevalence among physicians ( $\mathrm{n}=382$ )

\begin{tabular}{lccr}
\hline & Male & Female & \multicolumn{1}{c}{ Total } \\
\hline Total cancer & $9(5.2)$ & $21(10.0)$ & $30(7.9)$ \\
$\begin{array}{l}\text { Site of cancer } \\
\text { Thyroid }\end{array}$ & $2(1.2)$ & $11(5.2)$ & $13(3.4)$ \\
Breast & - & $5(2.4)$ & $5(1.3)$ \\
Stomach & $5(2.9)$ & $1(0.5)$ & $6(1.6)$ \\
Colon & $2(1.2)$ & $1(0.5)$ & $3(0.8)$ \\
Lung & - & $2(1.0)$ & $2(0.5)$ \\
Cervix & - & $2(1.0)$ & $2(0.5)$ \\
\hline
\end{tabular}

Values are presented as number (\%).

Therefore, a total of 31 cancers were validated in this study. Among 172 male physicians, 9 subjects $(5.2 \%)$ were diagnosed with cancer. The most common cancer among males was stomach cancer, with 5 cases (2.9\%), followed by 2 cases (1.2\%) of thyroid cancer and 2 cases (1.2\%) of colon cancer. Among female physicians, 21 (10.0\%) were diagnosed with cancer. The most common cancer among females was thyroid cancer with 11 cases $(5.2 \%)$, followed by 5 cases $(2.4 \%)$ of breast cancer, 2 cases (1.0\%) of lung cancer, 2 cases (1.0\%) of cervix cancer, 1 case $(0.5 \%)$ of stomach cancer, and 1 case $(0.5 \%)$ of colon cancer.

\section{Standardized Prevalence Ratio of Cancer}

We compared the prevalence of cancer among physicians to that of the general population with the same ethnicity and nationality based on data from the Korea National Cancer Incidence Database. ${ }^{21)}$ We calculated the SPR under the assumption that subjects who were identified as not having cancer based on their medical records and health screening results truly did not have any cancer in any part of the body (Table 3). We confirmed that physicians had a significantly higher prevalence of cancer compared to the general population. Among the 30 physicians in this study who were diagnosed with cancer, 13 (41.9\%) were newly diagnosed with cancer during the routine health assessment. The expected cancer prevalence among male physicians was found to be about 2.47 times higher than the expected prevalence of cancer among the general population $(\mathrm{P}=0.006)$. When the prevalence was compared according to cancer type, the prevalence of colon cancer was not significantly higher $(\mathrm{P}=0.124)$, but the prevalences of thyroid and gastric cancer were 7.15 times and 5.41 times higher, respectively, than that of the general Korean population $(\mathrm{P}=0.001$ and $\mathrm{P}<0.001$ ). Among female physicians, the expected cancer prevalence was 3.94 times higher than the expected prevalence among the general population $(\mathrm{P}<0.001)$. Although the prevalence of stomach and colon cancer was not significantly higher compared to the general population, we found an increased prevalence of thyroid cancer (SPR, 6.26; 95\% CI, 3.42 to 11.45), breast cancer (SPR, 4.60; 95\% CI, 1.09 to 11.13), lung cancer (SPR, 12.36; 95\% CI, 3.13 to 48.93), and cervix cancer (SPR, $16.52 ; 95 \% \mathrm{CI}, 4.20$ to 64.93$)$.
Table 3. SPR of cancers among physicians

\begin{tabular}{lcccr}
\hline & Observed & Expected & $\begin{array}{c}\text { SPR } \\
(95 \% \text { confidence interval })\end{array}$ & P-value \\
\hline Male $(\mathrm{n}=172)$ & & & & \\
$\quad$ Total cancer & 9 & 3.74 & $2.47(1.27-4.84)$ & 0.006 \\
Thyroid & 2 & 0.28 & $7.15(1.79-28.59)$ & 0.001 \\
Stomach & 5 & 0.94 & $5.41(2.23-13.11)$ & $<0.001$ \\
Colon & 2 & 0.71 & $2.84(0.71-11.42)$ & 0.124 \\
Female ( $\mathrm{n}=210)$ & & & & \\
Total cancer & 21 & 5.73 & $3.94(2.51-6.17)$ & $<0.001$ \\
Thyroid & 11 & 1.82 & $6.26(3.42-11.45)$ & $<0.001$ \\
Breast & 5 & 1.10 & $4.60(1.09-11.13)$ & $<0.001$ \\
Stomach & 1 & 0.58 & $1.72(0.24-12.26)$ & 0.582 \\
Colon & 1 & 0.58 & $1.71(0.24-12.18)$ & 0.586 \\
Lung & 2 & 0.16 & $12.36(3.13-48.93)$ & $<0.001$ \\
Cervix & 2 & 0.12 & $16.52(4.20-64.93)$ & $<0.001$ \\
\hline
\end{tabular}

SPR, standardized prevalence ratio.

\section{DISCUSSION}

For all cancers combined, the data from our study showed that SPRs for cancer among physicians were significantly higher than those of the general population in Korea. The SPR for male physicians was 2.47 (95\% CI, 1.27 to 4.84) and the SPR for female physicians was 3.94 (95\% CI, 2.51 to 61.7$)$. The SPRs for thyroid and stomach cancers tended to be higher among male physicians and the SPRs for breast, thyroid, lung, and cervix cancers were significantly higher among female physicians. These findings provide evidence that the cancer prevalence among both male and female physicians is higher than that in the general population in Korea.

Although physicians are usually expected to be healthier than the general population, several studies have reported higher cancer rates among physicians, similar to our study. ${ }^{13-15,23-25)}$ Mortality data of US radiology technologists showed that the risk of breast cancer was significantly higher than that in the general population of working women after adjusting for known risk factors such as family history of breast cancer, age of menopause, and number of live births (relative risk [RR], 2.92; $95 \% \mathrm{CI}, 1.22$ to 7.00$){ }^{13)}$ In this study, there was an increased risk of leukemia among radiology technologists. In Chinese cohort data, the risks for leukemia and cancers of the skin, female breast, lung, liver, bladder, and esophagus were significantly higher in 27,011 medical diagnostic X-ray workers. ${ }^{15)}$ In a study of US radiology workers, the melanoma risk was significantly elevated as much as 2.4 -fold among those who worked for 5 or more years as radiology technologists. ${ }^{14)}$ The risk was also modestly elevated among those who did not usually wear a lead apron or protective shield in the work place (RR, 1.4; 95\% CI, 0.8 to 2.5). More cases of squamous cell carcinoma of the skin caused by excessive radiation were reported in a study of radiologists in the UK. ${ }^{25)}$ Similar results regarding an increased risk of cancer among physicians were shown in studies of female orthopedic surgeons. ${ }^{23,24)}$ These studies suggest that the expectation that physicians are healthier than the general public is false, and the prevalence of cancer among physicians 
was reported to be higher compared to the general population. Questions remain regarding how we should interpret the higher prevalences of cancer among physicians, as well as the reason for such increases. The exact cause of these results has not yet been identified, and only some hypotheses have been considered.

In contrast to the expectation that a physician's medical knowledge and high socioeconomic status would help them maintain a healthy life, the reality is that their health may actually be threatened for several reasons. Although it varies according to sex and professional specialty, most physicians are routinely exposed to environmental factors that may increase the risk of cancer, such as excessive workload, irregular hours, and exposure to harmful chemical and physical agents within the medical environment. In reality, it is not easy to protect their health in light of these harmful environmental factors. The health of physicians is likely to be adversely affected as a result of this work environment.

Excessive workload and stress are thought to cause physical and mental health problems in physicians. Burnout syndrome is a complex, work-related syndrome with three characteristics: emotional exhaustion, depersonalization, and low personal accomplishment. It can have a negative impact on quality of life and cause compassion fatigue (an emotional and physical burden felt by those helping others in distress). ${ }^{23)}$ Recently, several studies have suggested that people who regularly work the night shift in hospitals have a higher risk of breast cancer compared to those who work during the day. ${ }^{26-28)}$ Therefore, it is necessary to consider the possibility that female physicians who frequently work at night due to night duty or who often come to the hospital at night in response to emergency calls or surgery may experience an increased risk of breast cancer.

In addition, physicians are part of an occupational group that is routinely exposed to radiation, which can increase the risk of cancer in many organs and tissues. Moreover, radiologic procedures, such as diagnostic, therapeutic, and interventional medicine procedures, are increasingly used in modern medicine as numerous new radiologic procedures are introduced. ${ }^{12)}$ In a nationwide cohort study in Finland, the risk of cancer in specific sites, particularly the risk of breast cancer, was found to be slightly increased in female physicians (standardized incidence ratio, 1.7; 95\% CI 1.0 to 3.1$){ }^{29)}$ In particular, orthopedic surgeons, neurosurgeons, interventional radiologists, and interventional cardiologists were more likely to be exposed to ionizing radiation. In urgent cases, physicians do not always wear protective shielding, such as a lead apron and thyroid shield. Moreover, there are no regulations or systematic education programs regarding the use of protective equipment to shield physicians from radiation in order to reduce the risk of skin cancer, leukemia, multiple myeloma, and thyroid cancer.

Generally, female physicians tend to have their first child later in life and are known to give birth to fewer children overall. ${ }^{26-28)}$ In some cases, they do not give birth at all. In addition, they can easily self-prescribe estrogen hormone agents that may increase the risk of breast cancer. ${ }^{30)}$ Several reproductive characteristics, including a short duration of breastfeeding and easy access to estrogen, may increase the risk of breast cancer among female physicians..$^{24,31)}$ For these reasons, female orthopedic surgeons in the United States have been found to have a three times higher prevalence of breast cancer than the general population of professional women. ${ }^{24)}$

Another possible reason for the increased cancer prevalence among physicians may be due to increased rates of obesity resulting from lifestyle changes. According to the Korean National Health and Nutrition Examination Survey (KNHANES) I (1998), II (2001), III (2005), and IV (2007-2009), the obesity rate was found to be $26.0 \%, 32.4 \%, 35.1 \%$, and $36.3 \%$, respectively, in Korean men over the age of 20 ( $P$ for trend $=0.006$, $\beta=0.018) .{ }^{32)}$ Our study found the obesity rate (45.5\%) in men to be higher than the KNHANES results. Therefore, the prevalence of colon, stomach, and thyroid cancers, which are known to be associated with obesity, appears to be higher than that in the general population. For these reasons, physicians themselves should check their health status, maintain healthy eating habits, and participate in regular exercise.

Although the results of this study are very significant, several limitations have to be taken into consideration when interpreting our results. Since this was a cross-sectional study, we could not determine a causal relationship to explain the higher SPRs for cancers among physicians. Moreover, bias may be present as a result of the subjects who participated, because only physicians who participated in a medical health check-up program at a single health promotion center in Korea between 2010 and 2013 were registered in this study. Physicians who were more concerned about their own health, who had a family history of cancer, or who had previously suspected cancer symptoms might have been more likely to register. Also, there is a possibility that the physicians who did not receive a health check-up may actually be healthier, or in contrast, may neglect their own health care. Therefore, we cannot rule out the possibility that the results of this study are slightly overestimated or underestimated. To address this issue, a large-scale study aimed at physicians across the country is needed in the future.

As for the increased risk of lung cancer among female physicians, the possibility that this is a chance finding cannot be ruled out, because lung cancer is a rare cancer. Therefore, there may be no relationship between rare cancers and working as a physician. Moreover, information on cancer risk factors, such as demographics, lifestyle, radiation exposure, family history of cancer, and reproductive history, was not considered, because this research was based on pre-collected medical information. Since the general population works in numerous occupations, the heterogeneity of subjects can influence the result. Nevertheless, it is necessary to investigate why the prevalence of cancer among physicians is higher than that in the general population in Korea.

In summary, to the best of our knowledge, this study is the first to explore cancer prevalence among physicians in Korea. Since this research is based on a single university hospital, it will be necessary to check the health of physicians more precisely in future large-scale research across several hospitals. Clarifying the role and influential characteristics related to working as a physician is likely to require more 
study. Also, it is necessary to consider developing educational programs to teach physicians about their own health care and management.

\section{CONFLICT OF INTEREST}

No potential conflict of interest relevant to this article was reported.

\section{REFERENCES}

1. Frank E. STUDENTJAMA. Physician health and patient care. JAMA 2004;291:637.

2. Frank E, Biola H, Burnett CA. Mortality rates and causes among U.S. physicians. Am J Prev Med 2000;19:155-9.

3. Frank E, Brogan DJ, Mokdad AH, Simoes EJ, Kahn HS, Greenberg RS. Health-related behaviors of women physicians vs other women in the United States. Arch Intern Med 1998;158:342-8.

4. Carpenter LM, Swerdlow AJ, Fear NT. Mortality of doctors in different specialties: findings from a cohort of 20000 NHS hospital consultants. Occup Environ Med 1997;54:388-95.

5. Alexander BH, Checkoway H, Nagahama SI, Domino KB. Cause-specific mortality risks of anesthesiologists. Anesthesiology 2000;93:92230.

6. Rimpela AH, Nurminen MM, Pulkkinen PO, Rimpela MK, Valkonen T. Mortality of doctors: do doctors benefit from their medical knowledge? Lancet 1987;1:84-6.

7. Aasland OG, Hem E, Haldorsen T, Ekeberg O. Mortality among Norwegian doctors 1960-2000. BMC Public Health 2011;11:173.

8. Ramachandran A, Snehalatha C, Yamuna A, Murugesan N. High prevalence of cardiometabolic risk factors among young physicians in India. J Assoc Physicians India 2008;56:17-20.

9. Kay MP, Mitchell GK, Del Mar CB. Doctors do not adequately look after their own physical health. Med J Aust 2004;181:368-70.

10. Frank E, Rimer BK, Brogan D, Elon L. U.S. Women Physicians' personal and clinical breast cancer screening practices. J Womens Health Gend Based Med 2000;9:791-801.

11. Silagy C. A view from the other side: a doctor's experience of having lymphoma. Aust Fam Physician 2001;30:547-9.

12. Yoshinaga S, Mabuchi K, Sigurdson AJ, Doody MM, Ron E. Cancer risks among radiologists and radiologic technologists: review of epidemiologic studies. Radiology 2004;233:313-21.

13. Mohan AK, Hauptmann M, Freedman DM, Ron E, Matanoski GM, Lubin JH, et al. Cancer and other causes of mortality among radiologic technologists in the United States. Int J Cancer 2003;103:259-67.

14. Freedman DM, Sigurdson A, Rao RS, Hauptmann M, Alexander B, Mohan A, et al. Risk of melanoma among radiologic technologists in the United States. Int J Cancer 2003;103:556-62.

15. Wang JX, Zhang LA, Li BX, Zhao YC, Wang ZQ, Zhang JY, et al. Cancer incidence and risk estimation among medical $\mathrm{x}$-ray workers in China, 1950-1995. Health Phys 2002;82:455-66.
16. Kang SH, Boo YJ, Lee JS, Han HJ, Jung CW, Kim CS. High occupational stress and low career satisfaction of Korean surgeons. J Korean Med Sci 2015;30:133-9.

17. Galam E, Komly V, Le Tourneur A, Jund J. Burnout among French GPs in training: a cross-sectional study. Br J Gen Pract 2013;63:e217-24.

18. Khawaja O, Petrone AB, Aleem S, Manzoor K, Gaziano JM, Djousse L. Sleep duration and risk of lung cancer in the physicians' health study. Zhongguo Fei Ai Za Zhi 2014;17:649-55.

19. Bellolio MF, Cabrera D, Sadosty AT, Hess EP, Campbell RL, Lohse CM, et al. Compassion fatigue is similar in emergency medicine residents compared to other medical and surgical specialties. West J Emerg Med 2014;15:629-35.

20. Percy CL, Miller BA, Gloeckler Ries LA. Effect of changes in cancer classification and the accuracy of cancer death certificates on trends in cancer mortality. Ann N Y Acad Sci 1990;609:87-97.

21. Jung KW, Won YJ, Kong HJ, Oh CM, Cho H, Lee DH, et al. Cancer statistics in Korea: incidence, mortality, survival, and prevalence in 2012. Cancer Res Treat 2015;47:127-41.

22. WHO Expert Consultation. Appropriate body-mass index for Asian populations and its implications for policy and intervention strategies. Lancet 2004;363:157-63.

23. Chou LB, Chandran S, Harris AH, Tung J, Butler LM. Increased breast cancer prevalence among female orthopedic surgeons. J Womens Health (Larchmt) 2012;21:683-9.

24. Chou LB, Cox CA, Tung JJ, Harris AH, Brooks-Terrell D, Sieh W. Prevalence of cancer in female orthopaedic surgeons in the United States. J Bone Joint Surg Am 2010;92:240-4.

25. Berrington A, Darby SC, Weiss HA, Doll R. 100 years of observation on British radiologists: mortality from cancer and other causes 18971997. Br J Radiol 2001;74:507-19.

26. Akerstedt T, Knutsson A, Narusyte J, Svedberg P, Kecklund G, Alexanderson K. Night work and breast cancer in women: a Swedish cohort study. BMJ Open 2015;5:e008127.

27. Hansen J, Lassen CF. Nested case-control study of night shift work and breast cancer risk among women in the Danish military. Occup Environ Med 2012;69:551-6.

28. Hansen J. Increased breast cancer risk among women who work predominantly at night. Epidemiology 2001;12:74-7.

29. Jartti P, Pukkala E, Uitti J, Auvinen A. Cancer incidence among physicians occupationally exposed to ionizing radiation in Finland. Scand J Work Environ Health 2006;32:368-73.

30. Willey SC, Cocilovo C. Screening and follow-up of the patient at high risk for breast cancer. Obstet Gynecol 2007;110:1404-16.

31. Laden F, Spiegelman D, Neas LM, Colditz GA, Hankinson SE, Manson JE, et al. Geographic variation in breast cancer incidence rates in a cohort of U.S. women. J Natl Cancer Inst 1997;89:1373-8.

32. Kang HT, Shim JY, Lee HR, Park BJ, Linton JA, Lee YJ. Trends in prevalence of overweight and obesity in Korean adults, 1998-2009: the Korean National Health and Nutrition Examination Survey. J Epidemiol 2014;24:109-16. 